\title{
Immunohistochemical Expression of Mast Cells Using c-Kit in Various Grades of Oral Submucous Fibrosis
}

\author{
Musarrat J. Khatri, ${ }^{1}$ Rajiv S. Desai, ${ }^{2}$ G. S. Mamatha, ${ }^{1}$ Meena Kulkarni, ${ }^{1}$ and Jay Khatri ${ }^{3}$ \\ ${ }^{1}$ Department of Oral Pathology, Dr. D. Y. Patil Dental College \& Hospital, Dr. D. Y. Patil Vidyapeeth, \\ Mahesh Nagar, Pimpri, Pune 411 018, India \\ ${ }^{2}$ Department of Oral Pathology, Nair Hospital Dental College, Mumbai 400 008, India \\ ${ }^{3}$ Department of Orthodontics, Dr. D. Y. Patil Dental College \& Hospital, Dr. D. Y. Patil Vidyapeeth, \\ Mahesh Nagar, Pimpri, Pune 411 018, India
}

Correspondence should be addressed to Musarrat J. Khatri; dr.musarratshaikh@gmail.com

Received 10 June 2013; Accepted 1 August 2013

Academic Editors: A. Gocht, C. K. Panda, and A. Wincewicz

Copyright (C) 2013 Musarrat J. Khatri et al. This is an open access article distributed under the Creative Commons Attribution License, which permits unrestricted use, distribution, and reproduction in any medium, provided the original work is properly cited.

Oral submucous fibrosis (OSF) is a high risk precancerous condition characterized by changes in the connective tissue fibers of lamina propria and deeper parts of mucosa. Mast cells are local residents of connective tissue and have been identified to participate in fibrotic process. These cells produce pharmacologically active substances necessary for the physiological function of our body in response to various stimuli as and when required and also play a significant role in the pathogenesis of oral diseases. Ten healthy volunteers and 30 clinically diagnosed OSF cases with histopathological confirmation were included in the study. Immunohistochemical (c-kit) as well as acidified toluidine blue staining techniques were used to evaluate density and expression of mast cells. The mast cell density assessed using c-kit and toluidine blue showed significant difference in various stages of OSF. In general the mean number of mast cells obtained using c-kit was found to be more than that obtained using toluidine blue in various stages of OSF. The comparison of mast cell densities using immunohistochemistry (c-kit) and toluidine blue stain confirmed that c-kit is a more reliable technique to assess mast cell density in OSF.

\section{Introduction}

Oral submucous fibrosis (OSF) is a chronic, progressive, and devastating disease which is prevalent in South East Asia. This is known to affect most parts of oral cavity, pharynx, and upper third of oesophagus. Although the pathogenesis of the disease is considered as multifactorial with areca nut chewing, ingestion of chilies, genetic and immunological processes, and nutritional deficiencies, areca nut is found to be the main aetiological factor for developing OSF [1]. In the initial stages, the disease is expressed clinically with burning sensation of mouth, blister formation, ulceration, excessive salivation, and defective gustatory sensation. As the disease progresses, the mucosa becomes blanched and fibrous bands appear vertically leading to difficulty in mouth opening [2]. Characteristic histopathological features of this disease include atrophic epithelium with an intercellular edema and moderate epithelial hyperplasia associated with progression of the disease.

Many studies have revealed that mast cells play an important role in OSF as they have been identified to participate in fibrotic process. The pathogenic mechanism in OSF begins in the connective tissue region and mast cells are the local residents of the connective tissue. Studies on mast cells in normal and various pathological conditions have revealed them to be complex, well-engineered, and multifunctional cells playing a central role in acquired and innate immunity [3]. Mast cells are known to produce pharmacologically active substances like histamine, heparin, proteolytic enzymes, platelet activating factor, leukotrienes, prostaglandins, and so forth in response to various stimuli as and when required. In OSF, a vascular response due to inflammation is commonly found and may be a reason for increased number of mast cells in earlier stages of the disease [4]. 
Differentiation of mast cells from fibroblasts using hematoxylin and eosin staining is difficult since mast cells have the same staining characteristics as the fibroblasts. Therefore $1 \%$ toluidine blue, a selective stain, is routinely used for staining of mast cells. This involves metachromatic staining of the mast cell granules. The mast cell granules are stained purplish red and nuclei appear sky blue in colour $[5,6]$. Recently immunohistochemical staining (c-kit) has been introduced as a special staining procedure for mast cells. In the immunohistochemical staining, the c-kit protooncogene encodes a transmembrane tyrosine kinase receptor, c-kit (CD117), which is closely related to the platelet derived growth factor family. This antibody recognizes the extracellular domain and is expressed by a variety of normal and abnormal cell types. In normal cells, the CD117 antibody has been shown to label breast epithelium, germ cells, melanocytes, stem cells, and mast cells. The c-kit ligand is an early haematopoietic growth factor and is known by various names, including kit ligand, steel factor, stem cell factor, and mast cell growth factor. By using this staining technique a strong membrane as well as cytoplasmic staining is evident in all mast cells and hence helpful in the diagnosis of mast cell diseases and mast cell tumours [7].

In the literature very less information is available on comparative account of staining techniques used for evaluating mast cell density in OSF. Therefore this study was designed to evaluate density and expression of mast cells in various stages of OSF with the help of toluidine blue and immunohistochemical (c-kit) staining.

\section{Materials and Method}

2.1. Cases. Histologically diagnosed 30 OSF cases and the control group of 10 cases without any habit from the outpatient department of the institute were included in the study. Clinical data and informed consent were taken from these patients. The inclusion criteria for OSF cases were blanching, presence of fibrous bands with loss of elasticity of the oral mucosa and difficulty to open mouth. The OSF cases based on mouth opening were classified according to Lai et al. [8] as stage 1 , stage 2 , stage 3 , and stage 4 [9]. The patients having OSF associated with malignancy or any other disease condition or taking any treatment (presently or in the past) were excluded from the study.

2.2. Tissue Specimens and Staining Techniques. Buccal mucosa was selected as the site for biopsy. Punch biopsies $(5 \mathrm{~mm})$ were performed from identical sites in study cases as well as in controls. The biopsy samples were immediately fixed in $10 \%$ neutral buffered formalin and routinely processed for histology. Formalin fixed and paraffin embedded tissues were cut into 5-micron thick sections and categorized in the 3 groups to undergo three staining procedures. For histological confirmation of the diagnosis of OSF the sections were stained according to the routine procedure with haematoxylin and eosin. After confirmation of OSF remaining two sections were stained with $1 \%$ toluidine blue and immunohistochemical staining (c-kit), respectively.
2.2.1. Immunohistochemical Staining. Immunohistochemical staining was carried out using one-step polymer technique (Dako, envision). Formalin fixed and paraffin embedded tissues were cut into 5-micron thick sections. These sections were deparaffinized through 3 changes of xylene for, 5 minutes each, followed by 2 changes of ethanol 100\%, 5 minutes each. The endogenous peroxidase activity was quenched by exposing the slides to freshly prepared $1 \% \mathrm{H}_{2} \mathrm{O}_{2}$ in methanol for 30 minutes. The sections were then rehydrated by passing the slides through graded alcohols: 1 change of 95\%, 1 change of $70 \%$, and 1 change of $50 \%$ ethanol, and then rinsed in distilled water. All changes were for 5 minutes. Sections were washed in Tris buffer saline (TBS) pH 7.4 (50 mM tris): 2 washes, 5 min each. Next wash was in TBS supplemented with $3 \%$ bovine serum for $30 \mathrm{~min}$. The primary antibody used was polyclonal rabbit anti-CD 117 antibody (Dako, Denmark). The solutions were diluted optimally in TBS with $3 \%$ bovine serum (dilution factor for CD117 is 1 in 400). The sections were then incubated for $1 \mathrm{hr}$ at $37^{\circ} \mathrm{C}$ with primary antibody. Alternate control sections were also incubated overnight, but without primary antibody. Slides were then washed in TBSx2, 3 min each. Polymer was added for $30 \mathrm{~min}$ at $37^{\circ} \mathrm{C}$. Slides were washed in TBSx2, 3 min each. ABC (avidin biotin complex) solution was applied to the slides and incubated for $30 \mathrm{~min}$ at $37^{\circ} \mathrm{C}$. Slides were then washed in TBSx3 for $2 \mathrm{~min}$ each followed by incubation in DAB for $7 \mathrm{~min}$. Alternatively $15 \mu \mathrm{L}$ of chromogen was added to $1 \mathrm{~mL}$ of buffer solution. Slides were washed in TBS for $1 \mathrm{~min}$. Counterstaining was carried out with hematoxylin for $10 \mathrm{sec}$. These slides were then dehydrated in 3 changes of $95 \%$ ethanol and 2 changes of absolute alcohol, cleared in 2 changes of xylene, and mounted in dibutyl phthalate xylene (DPX). Mast cells were identified with brown cytoplasmic as well as membrane staining.

2.2.2. Acidified Toluidine Blue Staining. The sections were deparaffinized in two changes of xylene and hydrated through two changes of alcohol, 5 minutes in each solution. Then the sections were kept in water for 5 minutes. The sections were then placed in a coupling jar containing toluidine blue stain for 30 minutes and then blotted carefully. They were then placed in absolute alcohol for 1 minute, cleared in xylene, and mounted on the slide using DPX. The granules of mast cells were stained purple and the rest of the section was stained blue.

2.3. Assessment of Mass Cell Density. The mast cell count was carried out on each slide including control stained for c-kit and toluidine blue, as previously grouped. All the cases were further divided into different stages of OSF by the criteria mentioned above. Density was assessed in areas showing the highest density of staining (hot spots) as determined by an initial scan at low magnification $(\times 100)$. Mast cells were counted from 5 different fields at 400x magnification in an image analyzer (Leica QWin V3 Software Image Analysis System) and the findings were recorded. For each slide, the number of mast cells from each field were counted, added up, and then divided by the number of fields to get the mean value. The statistical analysis was carried out using 


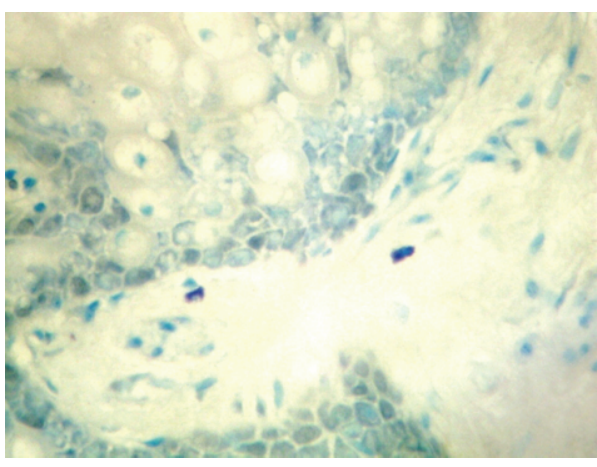

(a)

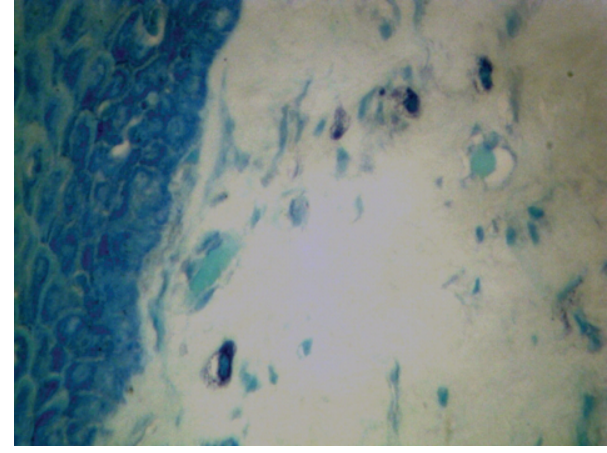

(b)

FIGURE 1: Detection of mast cells in (a) normal buccal mucosa and (b) oral submucous fibrosis using toluidine blue stain where the cells exhibit metachromasia.

TABLE 1: Correlation of mast cell density using toluidine blue and c-kit between control and various stages of OSF.

\begin{tabular}{lccccccccc}
\hline $\begin{array}{l}\text { Stages of OSF } \\
\text { and control }\end{array}$ & $\begin{array}{c}\text { Mean mast cell } \\
\text { density }\end{array}$ & $\begin{array}{c}\text { Mann-Whitney } \\
U \text { statistics }\end{array}$ & $P$ value & Significance & $\begin{array}{c}\text { Mean mast cell } \\
\text { density }\end{array}$ & $\begin{array}{c}\text { Mann-Whitney } \\
U \text { statistics }\end{array}$ & $P$ value & Significance \\
\hline Control & 2.18 & - & - & - & 4.26 & - & - & - \\
Stage 2 & 5.10 & 5.50 & 0.040 & Significant & 9.55 & 5.00 & 0.034 & Significant \\
Stage 3 & 3.09 & 64.00 & 0.28 & Nonsignificant & 9.08 & 15.50 & 0.00 & Significant \\
Stage 4 & 2.64 & 31.50 & 0.26 & Nonsignificant & 8.24 & 13.50 & 0.01 & Significant \\
\hline
\end{tabular}

Kruskal-Wallis test to compare mean values (cases, within cases and controls) and Mann-Whitney $U$ test for individual mean values.

\section{Results}

The findings of the study suggested that the age range of 30 cases with OSF was 15-55. From the total 30 cases, stage 3 OSF was prominently observed in 17 cases ( 15 male and 2 female) out of which eight cases were of age range 15-25. A marked gender difference was noted with overall male dominance in all age groups. There were no cases identified for OSF clinical stage 1.

Statistical analysis of mean mast cell densities assessed using toluidine blue (Figure 1) showed an increase in stage 2 as compared to control $(P<0.05)$. The results were statistically nonsignificant for stage 3 and stage 4 (Table 1 ). The results using c-kit in (Figure 2) showed an increase in cell densities among all OSF stages as compared to control (Table 1). Overall increase in cell densities was observed by using c-kit as compared to toluidine blue.

Furthermore the mean mast cell densities in different stages of OSF and control were compared. Statistical analysis suggested that comparison of the mast cell densities obtained using toluidine blue and c-kit was significant $(P<0.01)$ in control as well as stage 3 and stage 4 . However the results obtained for stage 2 OSF (Table 2) were not in accordance.

Within the group, no significant differences were observed in the mean mast cell densities by using both techniques (Table 3), although there was a naked eye difference in the number of mast cells, which were more obtained using c-kit.

\section{Discussion}

Oral submucous fibrosis (OSF) is a premalignant fibrotic condition. Several lines of evidence suggest that mast cells may participate in fibrotic process. It has been well established that oral squamous cell carcinoma (SCC) progresses in a multistep fashion: initially from normal epithelium, hyperkeratosis, premalignant dysplasia, and carcinoma in situ to invasive SCC. Angiogenesis as well as number of mast cells appeared to show a linear increase from normal oral mucosa, hyperkeratosis, and premalignant dysplasia to invasive SCC [10]. Studies on mast cells have noted that their number increases during initiation of OSF and abundant mast cells are observed in stage 1 and stage 2 of OSF $[3,11]$. These findings suggested that mast cells may upregulate angiogenesis in oral squamous cell carcinogenesis, perhaps via the release of mast cell tryptase. Thus number of mast cells may be used as indicator for disease progression.

Toluidine blue and immunohistochemical staining techniques are known to reliably identify mast cell granules. In this study, the mast cell density was assessed using both of these techniques. Our study group involved 30 cases of OSF and 10 controls. The mast cell density obtained using c-kit was higher in different stages of OSF as compared to normal mucosa. However the mean mast cell density assessed using toluidine blue for stage 2 was more as compared to control and was nonsignificant between stage 3 , stage 4 and control. 


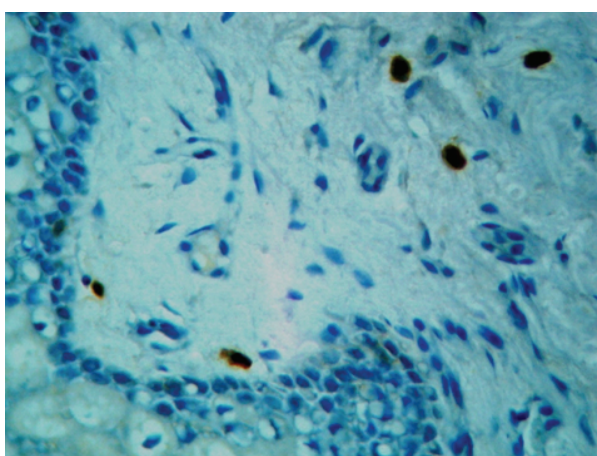

(a)

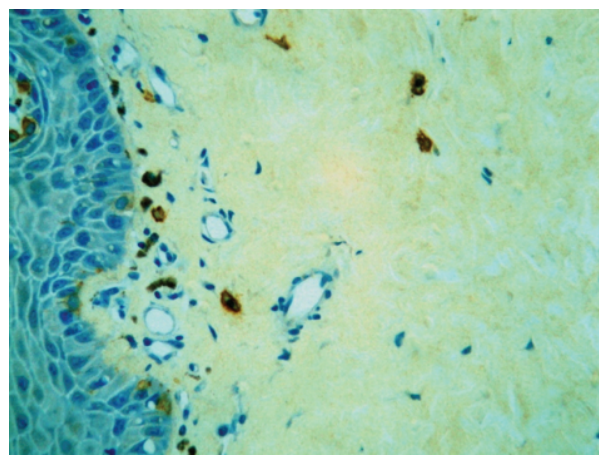

(c)

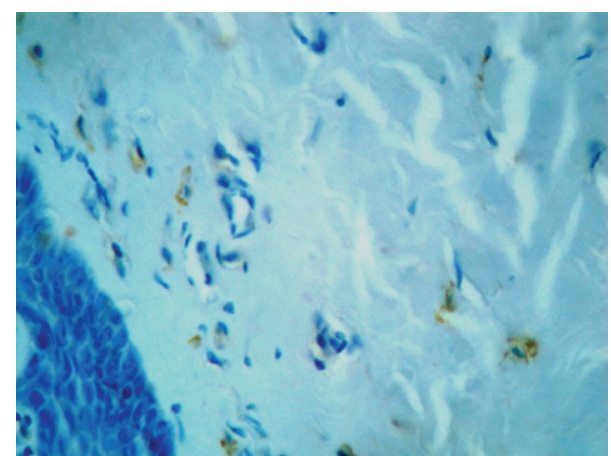

(b)

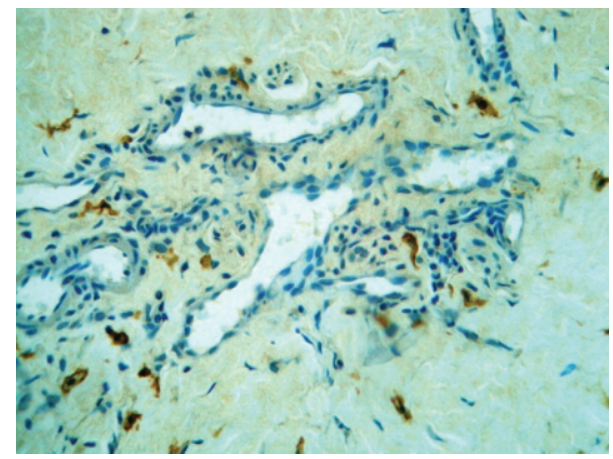

(d)

FIGURE 2: Detection of mast cells using immunohistochemical staining method (c-kit) in (a) normal buccal mucosa, (b) stage 2 OSF, (c) stage 3 OSF, and (d) stage 4 OSF.

TABLE 2: Correlation of mast cell density among c-kit and toluidine blue between control and various stages of OSF.

\begin{tabular}{|c|c|c|c|c|c|}
\hline \multirow{2}{*}{$\begin{array}{l}\text { Stages of OSF and } \\
\text { controls }\end{array}$} & \multicolumn{2}{|c|}{ Mean mast cell density } & \multirow{2}{*}{ Mann-Whitney $U$ statistics } & \multirow{2}{*}{$P$ value } & \multirow{2}{*}{ Significance } \\
\hline & c-kit & Toluidine blue & & & \\
\hline Control & 4.26 & 2.18 & 5.00 & 0.001 & Significant \\
\hline Stage 2 & 9.55 & 5.10 & 4.00 & 0.24 & Nonsignificant \\
\hline Stage 3 & 9.08 & 3.09 & 11.50 & 0.00 & Significant \\
\hline Stage 4 & 8.24 & 2.64 & 4.50 & 0.001 & Significant \\
\hline
\end{tabular}

TABLE 3: Correlation of mast cell density within group among various stages of OSF.

\begin{tabular}{|c|c|c|c|c|c|c|c|c|}
\hline \multirow{2}{*}{ Stages of OSF } & \multicolumn{4}{|c|}{ Using c-kit } & \multicolumn{4}{|c|}{ Using toluidine blue } \\
\hline & $\begin{array}{l}\text { Mean mast } \\
\text { cell density }\end{array}$ & Test statistics & $P$ value & Significance & $\begin{array}{l}\text { Mean mast cell } \\
\text { density }\end{array}$ & Test statistics & $P$ value & Significance \\
\hline Stage 2 & 9.55 & & & & 5.10 & & & \\
\hline Stage 3 & 9.08 & 0.51 & 0.775 & Nonsignificant & 3.09 & 3.02 & 0.22 & Nonsignificant \\
\hline Stage 4 & 8.24 & & & & 2.64 & & & \\
\hline
\end{tabular}

The comparison of mast cell density between control and various stages of OSF among c-kit and toluidine blue showed significant difference in the mean mast cell density in the control group. However, no significant difference in the mean mast cell density for stage 2 was shown. There was significant difference in the mean mast cell density for stage 3 as well as stage 4 as compared to control. These findings are against the previous study by Bhatt and Dholakia [11].
Comparison of the mean mast cell density within the group showed no significant difference using either c-kit or toluidine blue. Although there was visible difference in the mean mast cell density among the various stages of OSF using c-kit as well as toluidine blue, statistical analysis suggested the difference as nonsignificant. This indicates limitations of the test to detect the difference of interest using inadequate sample size. 
As the results suggested that the number of mast cells obtained using c-kit was definitely more than that obtained with toluidine blue, it was confirmed that the staining techniques play an important role in identifying number of mast cells in various stages of OSF. This can be attributed to difference in the staining principles of these two techniques. The immunohistochemical staining is based upon the principle of strong membrane (antigen-antibody reaction) as well as cytoplasmic staining while toluidine blue staining is based upon metachromatic staining principle. The mast cells contain metachromatic granules and their functional status is associated with cell degranulation. Thus degranulated mast cells are unlikely to be stained using toluidine blue. This technique may also stain cells such as macrophages and fibroblasts due to presence of released mast cell granules as a result of phagocytosis, and it may fail to stain immature mast cells as they may not contain these granules. In recent study by Rodini et al. it was shown that immunohistochemical technique is more specific than metachromatic staining [12]. Similarly during this study the mast cell densities obtained using c-kit were always higher as compared to toluidine blue suggesting specificity of the immunohistochemical staining. Thus the results confirm that c-kit is a more reliable technique for evaluating mast cell densities as well as their expression.

\section{Conclusion}

Our findings suggest that as the stage of OSF progresses from I to IV the number of mast cells increases and remains constant at that number but does not drop down in higher stages as against the results obtained using toluidine blue. The present study confirms c-kit to be more specific as well as reliable technique for expression of mast cells in OSF.

\section{Conflict of Interests}

The authors declare that they have no conflict of interests.

\section{Acknowledgment}

The authors thank Dr. Anita Borges for her kind support during the study period and Medilinkers Research Consultancy for their guidance in the preparation of the paper.

\section{References}

[1] P. Rajalalitha and S. Vali, "Molecular pathogenesis of oral submucous fibrosis-a collagen metabolic disorder," Journal of Oral Pathology and Medicine, vol. 34, no. 6, pp. 321-328, 2005.

[2] R. Rajendran, "Oral submucous fibrosis: etiology, pathogenesis, and future research," Bulletin of the World Health Organization, vol. 72, no. 6, pp. 985-996, 1994.

[3] M. R. Ankle, A. D. Kale, and R. Nayak, "Mast cells are increased in leukolpakia, oral submucous fibrosis, oral lichen planus and oral squamous cell carcinoma," Journal of Oral and Maxillofacial Pathology, vol. 11, pp. 18-22, 2007.

[4] S. M. Sirsat and J. J. Pindborg, "The vascular response in early and advanced oral submucous fibrosis," Acta Pathologica et Microbiologica Scandinavica, vol. 70, no. 2, pp. 179-184, 1967.
[5] J. Janes and J. R. McDonald, "Mast cells-their distribution in various human tissues," Archives of Pathology \& Laboratory Medicine, vol. 45, pp. 622-634, 1948.

[6] S. A. Shukla, R. Veerappan, J. S. Whittimore, L. Ellen Miller, and G. A. Youngberg, "Mast cell ultrastructure and staining in tissue," Methods in Molecular Biology, vol. 315, pp. 63-76, 2006.

[7] D. A. Arber, R. Tamayo, and L. M. Weiss, "Paraffin section detection of the c-kit gene product (cd117) in human tissues: value in the diagnosis of mast cell disorders," Human Pathology, vol. 29, no. 5, pp. 498-504, 1998.

[8] D. R. Lai, H. R. Chen, Y. L. Huang, and C. C. Tsai, "Clinical evaluation of different treatment methods for oral submucous fibrosis: A 10-year experience with 150 cases," Journal of Oral Pathology and Medicine, vol. 24, pp. 402-406, 1995.

[9] K. Ranganathan and G. Mishra, "An overview of classification schemes for oral submucous fibrosis," Journal of Oral and Maxillofacial Pathology, vol. 10, pp. 55-58, 2006.

[10] A. Iamaroon, S. Pongsiriwet, S. Jittidecharaks, K. Pattanaporn, S. Prapayasatok, and S. Wanachantararak, "Increase of mast cells and tumor angiogenesis in oral squamous cell carcinoma," Journal of Oral Pathology and Medicine, vol. 32, no. 4, pp. 195199, 2003.

[11] A. P. Bhatt and H. M. Dholakia, "Mast cell density in oral submucous fibrosis," Journal of Indian Dental Association, vol. 49, pp. 187-191, 1977.

[12] C. D. O. Rodini, A. C. Batista, and V. S. Lara, "Comparative immunohistochemical study of the presence of mast cells in apical granulomas and periapical cysts: possible role of mast cells in the course of human periapical lesions," Oral Surgery, Oral Medicine, Oral Pathology, Oral Radiology, and Endodontics, vol. 97, no. 1, pp. 59-63, 2004. 


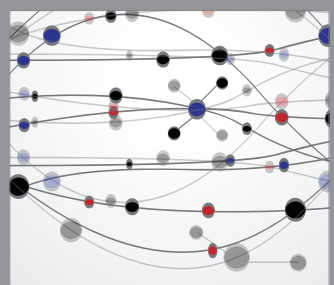

The Scientific World Journal
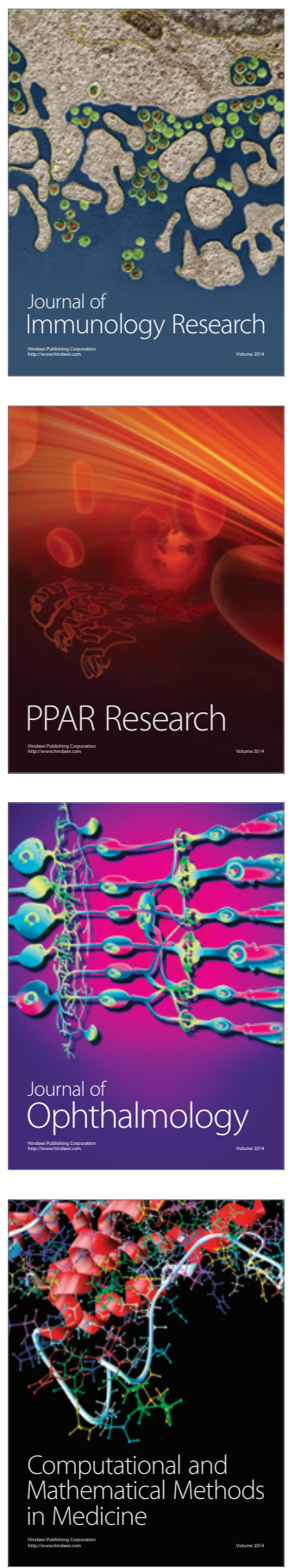

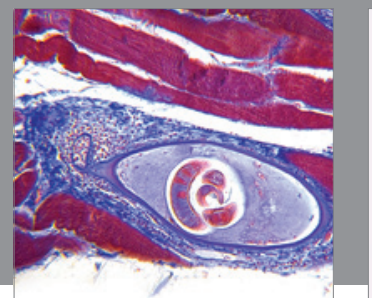

Gastroenterology

Research and Practice
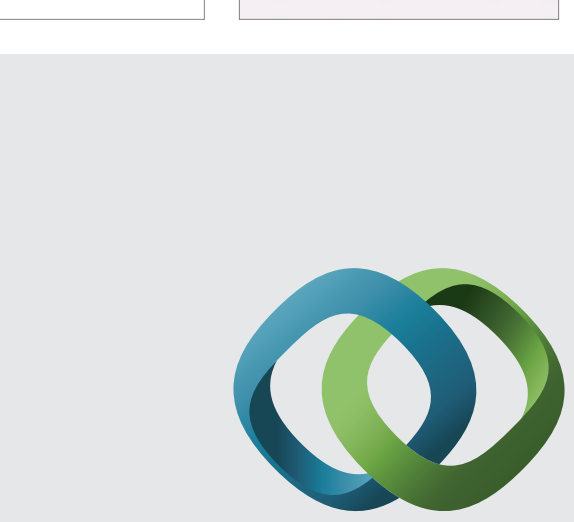

\section{Hindawi}

Submit your manuscripts at

http://www.hindawi.com
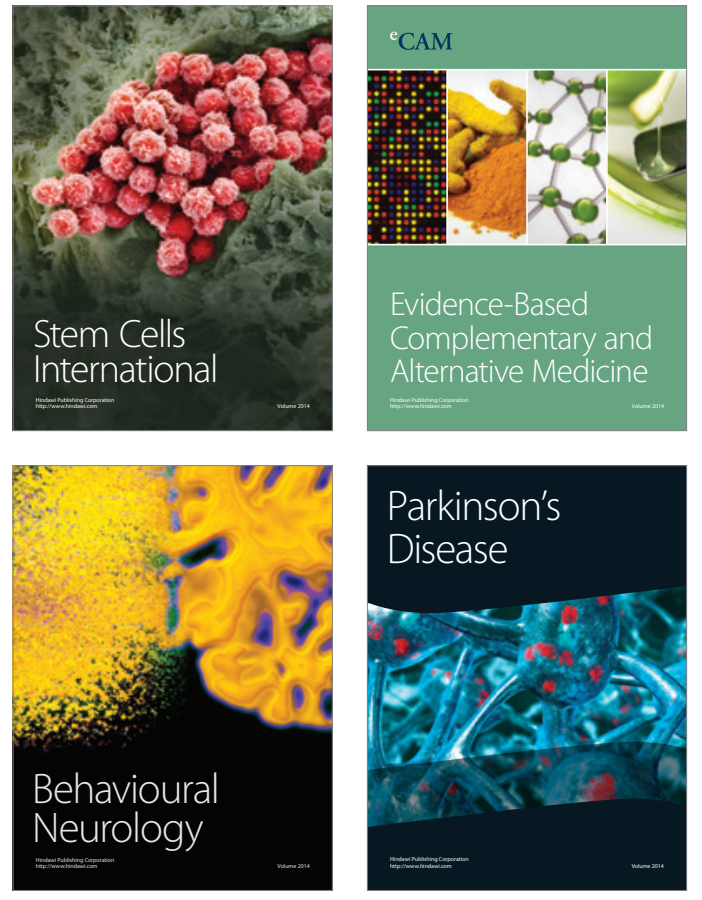
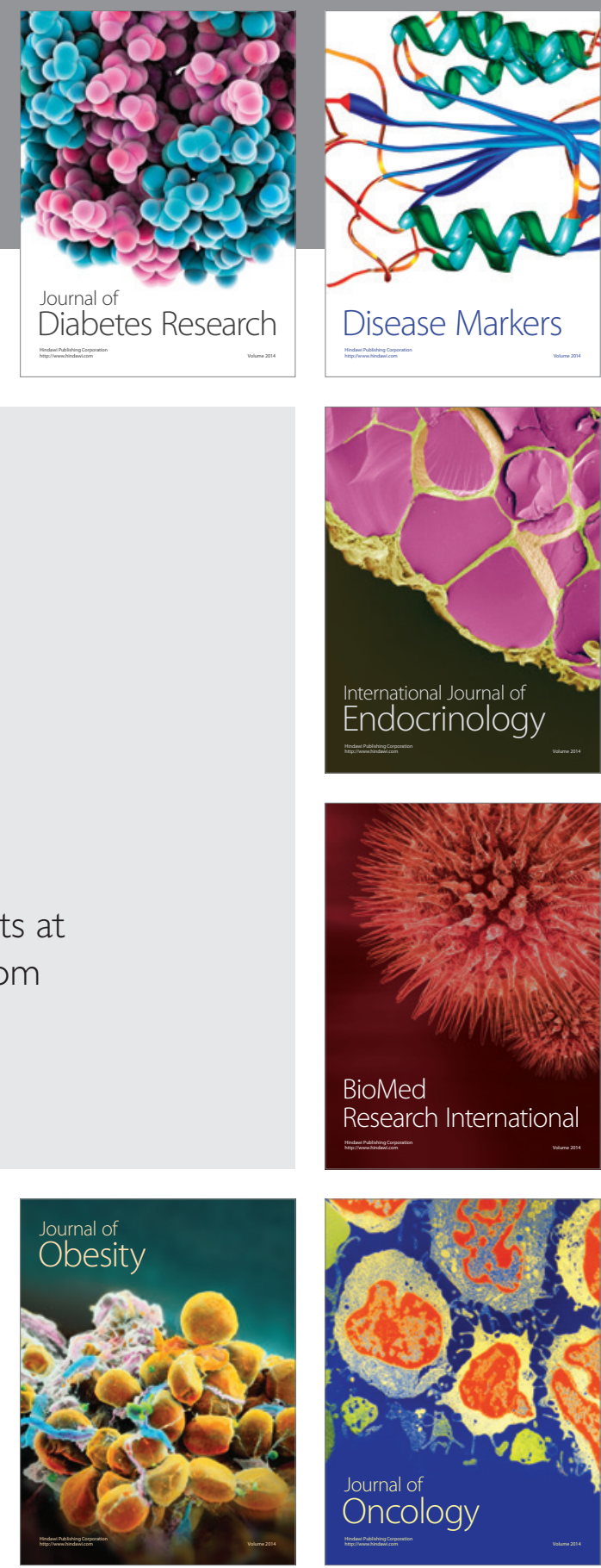

Disease Markers
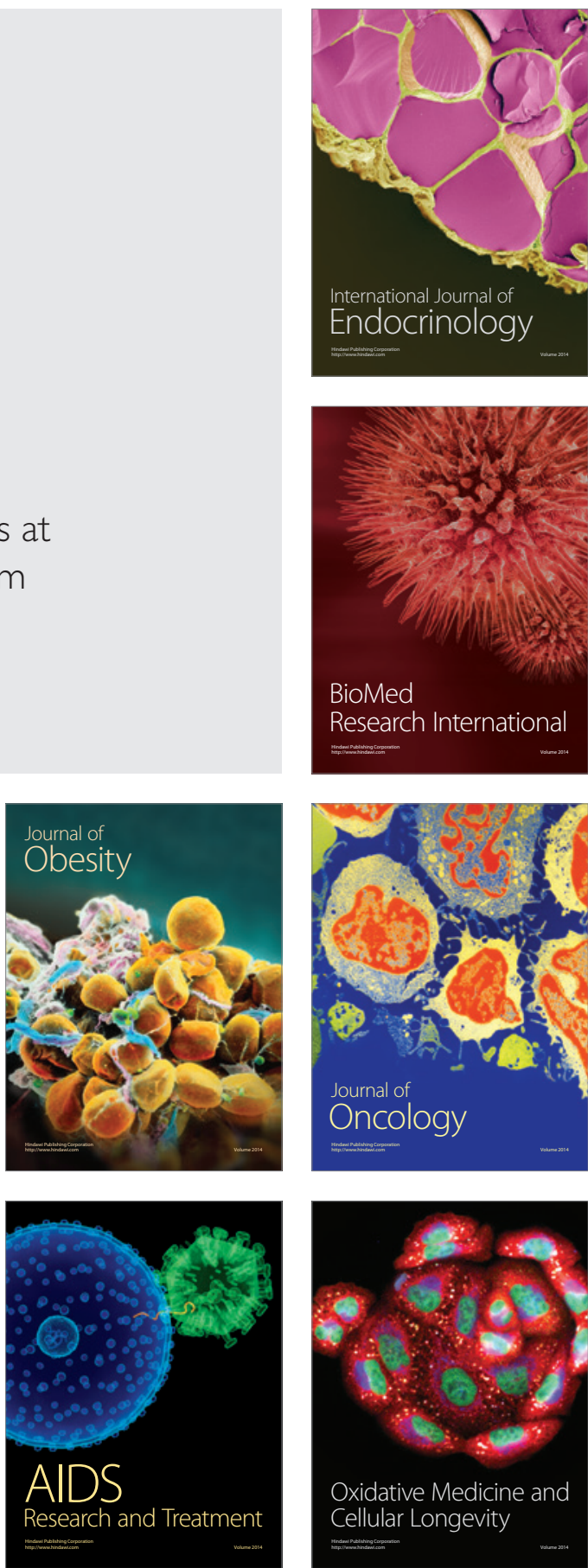